PROCEEDINGS OF THE

AMERICAN MATHEMATICAL SOCIETY

Volume 127, Number 7, Pages 2007-2012

S 0002-9939(99)04669-9

Article electronically published on February 26, 1999

\title{
A TRACE FORMULA FOR HANKEL OPERATORS
}

\author{
AURELIAN GHEONDEA AND RAIMUND J. OBER
}

(Communicated by Theodore W. Gamelin)

\begin{abstract}
We show that if $G$ is an operator valued analytic function in the open right half plane such that the Hankel operator $H_{G}$ with symbol $G$ is of trace-class, then $G$ has continuous extension to the imaginary axis,$$
G(\infty):=\lim _{\substack{r \rightarrow \infty \\ r \in \mathbb{R}}} G(r)
$$

exists in the trace-class norm, and $\operatorname{tr}\left(H_{G}\right)=\frac{1}{2} \operatorname{tr}(G(0)-G(\infty))$.
\end{abstract}

\section{INTRODUCTION}

Let $G$ be a scalar real-rational function whose poles are in the open left half plane and such that $G(\infty)=0$. In [1], [4], [5] it was shown that

$$
G(0)=2 \sum_{i=1}^{n} \lambda_{i}
$$

where $\lambda_{1}, \lambda_{2}, \ldots, \lambda_{n}$ are the eigenvalues, counted with their multiplicities, of the Hankel operator with symbol $G$. In [7] it was shown that a generalization of this result is possible in case the symbol is a non-rational Stieltjes function whose associated Hankel operator is of trace class. The purpose of this paper is to show that if $G$ is an operator valued analytic function in the open right half plane such that the Hankel operator $H_{G}$ with symbol $G$ is of trace-class, then $G$ has a continuous extension to the imaginary axis,

$$
G(\infty):=\lim _{\substack{r \rightarrow \infty \\ r \in \mathbb{R}}} G(r)
$$

exists in the trace-class norm, and $\operatorname{tr}(G(0)-G(\infty))=2 \operatorname{tr}\left(H_{G}\right)$. The existing results are special cases of the result that is presented in this paper. None of the existing proofs for the special cases seem to generalize directly to our situation. Therefore a new approach had to be introduced here. Common, however, with the methods of proof for the earlier results is that our proof was inspired by system theoretic methods.

We will assume throughout this paper that all Hilbert spaces are separable. This assumption is not a limitation, as most of the operators in this paper will be assumed compact.

Received by the editors May 29, 1997 and, in revised form, September 10, 1997.

1991 Mathematics Subject Classification. Primary 47B35; Secondary 47A56, 93B28.

This research was supported in part by NSF grant DMS-9501223.

(C)1999 American Mathematical Society 


\section{Continuous-time transfer Functions WITH TRACE-CLASS HANKEL OPERATORS}

Let $G$ be a function analytic in the open right half plane $\mathbb{C}_{+}=\{\lambda \in \mathbb{C} \mid \operatorname{Re}(\lambda)>$ $0\}$ with values in $\mathcal{L}(\mathcal{U}, \mathcal{Y})$, where $\mathcal{U}$ and $\mathcal{Y}$ are Hilbert spaces. Here $\mathcal{L}(\mathcal{U}, \mathcal{Y})$ denotes the space of bounded linear operators from $\mathcal{U}$ to $\mathcal{Y}$. Recall that the Hardy space $H_{\mathcal{Y}}^{2}\left(\mathbb{C}_{+}\right)$is naturally identified with a closed subspace of $L_{\mathcal{Y}}^{2}(i \mathbb{R})$ and, modulo this identification, let $P_{+}$denote the orthogonal projection of $L_{\mathcal{Y}}^{2}(i \mathbb{R})$ onto $H_{\mathcal{Y}}^{2}\left(\mathbb{C}_{+}\right)$. By definition, the Hankel operator with symbol $G$ is the operator

$$
H_{G}: \operatorname{Dom}\left(H_{G}\right) \rightarrow H_{\mathcal{U}}^{2}\left(\mathbb{C}_{+}\right), \quad f \mapsto P_{+} M_{G} R f,
$$

where $(R f)(\lambda)=f(-\lambda)$ for $\lambda \in \mathbb{C}_{+}$and $f$ rational in $H_{\mathcal{U}}^{2}\left(\mathbb{C}_{+}\right), M_{G}$ is the operator of multiplication with $G$, and

$$
\begin{array}{r}
\operatorname{Dom}\left(H_{G}\right)=\left\{f \in H_{\mathcal{U}}^{2}\left(\mathbb{C}_{+}\right) \mid f \text { rational, } G R f \text { has horizontal limit a.e. on } i \mathbb{R}\right. \\
\text { and the limit function is in } \left.L_{\mathcal{Y}}^{2}(i \mathbb{R})\right\} .
\end{array}
$$

We refer to [8] for the theory of Hankel operators and their applications. In this paper we will be mainly interested in the case when $H_{G}$ is everywhere defined on $\mathcal{H}_{\mathcal{U}}^{2}\left(\mathbb{C}_{+}\right)$and it is a bounded operator $H_{\mathcal{U}}^{2}\left(\mathbb{C}_{+}\right) \rightarrow H_{\mathcal{Y}}^{2}\left(\mathbb{C}_{+}\right)$.

Let $G_{c}$ be an analytic $\mathcal{L}(\mathcal{U}, \mathcal{Y})$-valued function in the open right half plane $\mathbb{C}_{+}$, where $\mathcal{U}$ and $\mathcal{Y}$ are Hilbert spaces. We associate to this "continuous-time transfer function" $G_{c}$ a "discrete-time transfer function" $G_{d}$ by (see e.g. [6])

$$
G_{d}(z):=G_{c}\left(\frac{z-1}{z+1}\right), \quad|z|>1 .
$$

Note that the function $G_{d}$, which clearly is analytic outside the unit disk, is also analytic at $\infty$.

Further, one can define another operator valued analytic function $g_{d}: \mathbb{D} \rightarrow$ $\mathcal{L}(\mathcal{U}, \mathcal{Y})$ by

$$
g_{d}(z)=\frac{1}{z}\left(G_{d}\left(\frac{1}{z}\right)-G_{d}(\infty)\right), \quad|z|<1 .
$$

The symbols $\mathbb{D}$ and $\mathbb{T}$ stand for the open unit disk $\{z \in \mathbb{C}|| z \mid<1\}$ and, respectively, the complex unit circle $\{z \in \mathbb{C}|| z \mid=1\}$. Associated with the function $g_{d}$ is the Hankel operator $H_{g_{d}}$. It is defined by

$$
H_{g_{d}} f=P_{+} M_{g_{d}} J f, \quad f \in \operatorname{Dom}\left(H_{g_{d}}\right),
$$

where $(J f)(z)=f(1 / z)$ for all rational $f$ in $H_{\mathcal{U}}^{2}(\mathbb{D})$ and $z \in \mathbb{D}, M_{g_{d}}$ denotes the multiplication operator with $g_{d}, P_{+}$denotes the orthogonal projection of $L_{\mathcal{Y}}^{2}(\mathbb{T})$ onto $H_{\mathcal{Y}}^{2}(\mathbb{D})$, and

$$
\begin{array}{r}
\operatorname{Dom}\left(H_{g_{d}}\right)=\left\{f \in H_{\mathcal{U}}^{2}(\mathbb{D}) \mid f \text { rational, } g_{d} J f \text { has radial limit a.e. on } i \mathbb{R}\right. \\
\text { and the limit function is in } \left.L_{\mathcal{Y}}^{2}(\mathbb{T})\right\} .
\end{array}
$$

The function $g_{d}$ has the Taylor expansion on $\mathbb{D}$

$$
g_{d}(z)=\sum_{k \geq 0} S_{k} z^{k}, \quad|z|<1
$$

where $S_{k} \in \mathcal{L}(\mathcal{U}, \mathcal{Y}), k \geq 0$. This series is absolutely and uniformly convergent on all compact subsets of $\mathbb{D}$. It is easy to see that the operator $H_{g_{d}}$ has the block-matrix 
Hankel representation

$$
H_{g_{d}}=\left[\begin{array}{cccccc}
S_{0} & S_{1} & S_{2} & \ldots & S_{k} & \ldots \\
S_{1} & S_{2} & S_{3} & \ldots & S_{k+1} & \ldots \\
S_{2} & S_{3} & \ldots & & & \\
\vdots & & & & & \\
S_{k} & & & & & \\
\vdots & & & & &
\end{array}\right]
$$

which acts at least formally on $\ell_{\mathcal{U}}^{2}$ and has values in $\ell_{\mathcal{Y}}^{2}$. The operators $S_{k} \in \mathcal{L}(\mathcal{U}, \mathcal{Y})$ are defined as in $(2.5)$. Here the canonical identification of $H_{\mathcal{U}}^{2}(\mathbb{D})\left(H_{\mathcal{Y}}^{2}(\mathbb{D})\right)$ with $\ell_{\mathcal{U}}^{2}\left(\ell_{\mathcal{Y}}^{2}\right)$ has been used, where $\ell_{\mathcal{U}}^{2}\left(\ell_{\mathcal{Y}}^{2}\right)$ is the Hilbert space of square summable sequences of vectors in $\mathcal{U}(\mathcal{Y})$.

Let us recall (e.g. see [8]) that, for a Hilbert space $\mathcal{H}$ there exists a natural unitary identification of the Hilbert spaces $H_{\mathcal{H}}^{2}(\mathbb{D})$ and $H_{\mathcal{H}}^{2}\left(\mathbb{C}_{+}\right)$given by

$$
V_{\mathcal{H}}: H_{\mathcal{H}}^{2}(\mathbb{D}) \rightarrow H_{\mathcal{H}}^{2}\left(\mathbb{C}_{+}\right), \quad f \mapsto\left(V_{\mathcal{H}} f\right)(\cdot)=\frac{1}{\sqrt{\pi}(1+\cdot)} f\left(\frac{1-\cdot}{1+\cdot}\right) .
$$

Coming back to our situation, just from the definitions (2.1), (2.4) and (2.7), a straightforward verification (e.g. see Theorem 4.6 in [8]) shows that

$$
H_{G_{c}} V_{\mathcal{U}}=V_{\mathcal{Y}} H_{g_{d}} .
$$

In the following, for a Hilbert space $\mathcal{H}$, we denote by $\mathfrak{S}_{1}(\mathcal{H})$ the ideal of all traceclass operators on $\mathcal{H}$. The trace of an arbitrary operator $T \in \mathcal{L}(\mathcal{H})$ is denoted by $\operatorname{tr}(T)$ and the trace-class norm is $\|T\|_{1}=\operatorname{tr}(|T|)$, where $|T|=\left(T^{*} T\right)^{1 / 2}$. For standard results on compact and trace-class operators we refer to [2].

Theorem 2.1. Let $\mathcal{U}$ be a Hilbert space. Let $G_{c}$ be an analytic $\mathcal{L}(\mathcal{U})$-valued function in the open right half plane $\mathbb{C}_{+}$. Assume that the Hankel operator $H_{G_{c}}$ with symbol $G_{c}$ is of trace-class. Then $G_{c}$ admits a $\|\cdot\|_{1}$-continuous extension to the imaginary axis $i \mathbb{R}$, including at $\pm i \infty$. In particular, the limit

$$
G_{c}(\infty):=\lim _{\substack{r \rightarrow+\infty \\ r \in \mathbb{R}}} G_{c}(r)
$$

exists in the norm $\|\cdot\|_{1}$, the operators $G_{c}(0)$ and $G_{c}(\infty)$ are nuclear, and

$$
\operatorname{tr}\left(H_{G_{c}}\right)=\frac{1}{2} \operatorname{tr}\left(G_{c}(0)-G_{c}(\infty)\right)
$$

Proof. Associated to the function $G_{c}$, we consider the functions $G_{d}$ and $g_{d}$ as in (2.2) and (2.3), respectively, with $\mathcal{U}=\mathcal{Y}$. We make use of (2.8) to see that the Hankel operator $H_{G_{c}}$ acting on $H_{\mathcal{U}}^{2}\left(\mathbb{C}_{+}\right)$is unitarily equivalent with the Hankel operator $H_{g_{d}}$ acting in $H_{\mathcal{U}}^{2}(\mathbb{D})$. Hence, the block matrix Hankel operator defined as in (2.6) is bounded in $\ell_{\mathcal{U}}^{2}$ and is a trace-class operator.

We consider now the sequence of mutually orthogonal selfadjoint projections $\left\{P_{k}\right\}_{k \geq 0} \in \mathcal{L}\left(\ell_{\mathcal{U}}^{2}\right)$, where $P_{k}$ is the orthogonal projection from $\ell_{\mathcal{U}}^{2}$ onto its $k$-th component (which we identify with $\mathcal{U}$ ). Then (see e.g. Theorem III.8.7 in [2]) we 
have that the diagonal block-matrix operator

$$
\sum_{k \geq 0} P_{k} H_{g_{d}} P_{k}=\left[\begin{array}{ccccc}
S_{0} & 0 & 0 & 0 & \cdots \\
0 & S_{2} & 0 & 0 & \cdots \\
& & \ddots & & \\
0 & \cdots & & S_{2 k} & \\
\vdots & & & & \ddots
\end{array}\right]
$$

is of trace-class and

$$
\left\|\sum_{k \geq 0} P_{k} H_{g_{d}} P_{k}\right\|_{1}=\sum_{k \geq 0}\left\|S_{2 k}\right\|_{1} \leq\left\|H_{g_{d}}\right\|_{1} .
$$

Moreover, all the operators $S_{2 k}, k=0,1, \ldots$, are of trace-class and

$$
\operatorname{tr}\left(H_{g_{d}}\right)=\operatorname{tr}\left(\sum_{k \geq 0} P_{k} H_{g_{d}} P_{k}\right)=\sum_{n=0}^{\infty} \operatorname{tr}\left(S_{2 n}\right) .
$$

Note that the sum (2.12) converges absolutely. Also recall (see e.g. [2]) that

$$
\left|\operatorname{tr}\left(T H_{g_{d}}\right)\right| \leq\|T\|\left\|H_{g_{d}}\right\|_{1}
$$

for any bounded operator $T$ with uniform (operator) norm $\|T\|$. If in the above inequality $T$ is the left shift on $\ell_{\mathcal{U}}^{2}$, it follows that the shifted Hankel operator

$$
H_{s}=T H_{g_{d}}=\left[\begin{array}{ccccc}
S_{1} & S_{2} & S_{3} & S_{4} & \cdots \\
S_{2} & S_{3} & S_{4} & \cdots & \\
S_{3} & S_{4} & \cdots & & \\
S_{4} & \cdots & & & \\
\vdots & & & &
\end{array}\right]
$$

is also of trace-class, since the shift operator is bounded and $H_{g_{d}}$ is of trace-class. As before, we obtain that

$$
\left\|\sum_{k \geq 0} P_{k} H_{s} P_{k}\right\|_{1}=\sum_{k \geq 0}\left\|S_{2 k+1}\right\|_{1} \leq\left\|H_{s}\right\|_{1} .
$$

Therefore, all the operators $S_{2 k+1}, k=0,1, \ldots$, are of trace-class and

$$
\operatorname{tr}\left(H_{s}\right)=\operatorname{tr}\left(\sum_{k \geq 0} P_{k} H_{s} P_{k}\right)=\sum_{k=0}^{\infty} \operatorname{tr}\left(S_{2 k+1}\right),
$$

where the sum converges absolutely.

From (2.11) and (2.13) it follows that

$$
\sum_{k \geq 0}\left\|S_{k}\right\|_{1}<\infty
$$

which is an operator version of a result by M. Rosenblum ([3]). Hence the Taylor series (2.3) of the function $g_{d}$ converges in the $\|\cdot\|_{1}$-norm for all $|z| \leq 1$. Note that, since all the operators $S_{n}, n=0,1, \ldots$, are of trace-class, $g_{d}(z)$ is also of trace-class for all $z \in \mathbb{D}$. Therefore, $g_{d}$ has a $\|\cdot\|_{1}$-continuous extension to the unit circle $\mathbb{T}$. Recovering $G_{c}$ from (2.2) and (2.3), we get

$$
G_{c}(\lambda)=\frac{1-\lambda}{1+\lambda} g_{d}\left(\frac{1-\lambda}{1+\lambda}\right)+g_{d}(0), \quad \operatorname{Re}(\lambda)>0 .
$$


Thus, modulo the multiplication with a scalar function and the addition of the trace-class operator $g_{d}(0)=S_{0}, G_{c}$ is obtained from $g_{d}$ by a conformal mapping of the unit disk $\mathbb{D}$ onto the open right half plane $\mathbb{C}_{+}$such that the unit circle $\mathbb{T}$ is mapped into the imaginary axis $i \mathbb{R}$. Hence $G_{c}$ has a $\|\cdot\|_{1}$-continuous extension on $i \mathbb{R}$, including at $\pm i \infty$. In particular, the limit (2.9) exists in the $\|\cdot\|_{1}$-norm and the operator $G_{c}(\infty)$ is of trace-class.

Furthermore, the values $g_{d}(1)$ and $g_{d}(-1)$ exist and are given by

$$
g_{d}(1)=\sum_{k=0}^{\infty} S_{k}, \quad g_{d}(-1)=\sum_{k=0}^{\infty}(-1)^{k} S_{k},
$$

where the sums converge absolutely and uniformly in the norm $\|\cdot\|_{1}$. From here and (2.12) we immediately have that

$$
\operatorname{tr}\left(H_{g_{d}}\right)=\sum_{n=0}^{\infty} \operatorname{tr}\left(S_{2 n}\right)=\frac{1}{2} \operatorname{tr}\left(g_{d}(1)+g_{d}(-1)\right) .
$$

From (2.14) it follows that

$$
G_{c}(0)=g_{d}(0)+g_{d}(1), \quad G_{c}(\infty)=g_{d}(0)-g_{d}(-1),
$$

which by subtraction yields

$$
G_{c}(0)-G_{c}(\infty)=g_{d}(1)+g_{d}(-1) .
$$

Finally, from (2.15) and (2.16) we obtain the desired formula (2.10).

The celebrated theorem of V.B. Lidskini states that the trace of a trace-class operator $T$ is given by

$$
\operatorname{tr}(T)=\sum_{n \geq 0} \lambda_{n}
$$

where $\left\{\lambda_{n}\right\}_{n \geq 0}$ is the sequence of its eigenvalues, counted according to their muliplicities. Therefore, if $\left\{\lambda_{n}\right\}_{n \geq 0}$ denotes the sequence of the eigenvalues, counted with their multiplicities, of the trace-class Hankel operator $H_{G_{c}}$ as in Theorem 2.1 then

$$
\operatorname{tr}\left(G_{c}(0)-G_{c}(\infty)\right)=2 \sum_{k \geq 0} \lambda_{k}
$$

This shows that Theorem 2.1 is a generalization of the previous results as in [1], [4], [5] (see (1.1)).

\section{REFERENCES}

1. K.V. Fernando, H. Nicholson, On the structure of balanced and other principal representations of SISO systems, IEEE Transactions on Automatic Control, 28(1983), 228-231. MR 84i:93028

2. I.C. Gohberg, M.G. Krein, Introduction to the Theory of Linear Nonselfadjoint Operators, Transl. Math. Monographs, Vol. 18, Amer. Math. Soc., Providence RI 1969. MR 39:7447

3. J.S. Howland, Trace class Hankel operators, Quart. J. Math. Oxford, 22(1971), 147-159. MR 44:5826

4. S.S. Mahil, F.W. Fairman, B.S. Lee, Some integral properties for balanced realizations of scalar systems, IEEE Transactions on Automatic Control, 29(1984), 181-183. MR 85b:93016

5. R.J. Ober, Balanced parametrization of classes of linear systems, SIAM Journal on Control and Optimization, 29(1991), 1251-1287. MR 92j:93028

6. R.J. Ober, S. Montgomery-Smith, Bilinear transformation of infinite-dimensional state-space systems and balanced realizations of nonrational transfer functions, SIAM Journal on Control and Optimization, 28(1990), 438-465. MR 91d:93019 
7. R.J. Ober, On Stieltjes functions and Hankel operators, Systems and Control Letters, 27(1996), 275-277. MR 97a:93034

8. J.R. Partington, An introduction to Hankel operators, Cambridge University Press, 1988. MR 90c: 47047

Institutul de Matematică Al Academiei romÂne, C.P. 1-764, 70700 Bucureşti, România

E-mail address: gheondea@imar.ro

Center for Engineering Mathematics EC35, University of Texas at Dallas, RichardSON, TeXas 75083-0688

E-mail address: ober@utdallas.edu 